\title{
Short-Term Load Forecasting for Electrical Dispatcher of Baghdad City based on SVM-FA
}

\author{
Aqeel S. Jaber ${ }^{1}$, Kosay A. Satar ${ }^{2}$, Nadheer A. Shalash ${ }^{3}$ \\ Department of Electrical Power Engineering Techniques \\ Al-Mamoun university college \\ Baghdad, Iraq
}

\begin{abstract}
The improvement of load forecasting accuracy is an important issue in the scientific optimization of power systems. The availability of accurate statistical data and a suitable scientific method are necessary for a perfect prediction of future occurrences. This research deals with the use of a regression forecast model (Support Vector Machine, SVM) for the prediction of the vector data for electrical power loading and temperature in Baghdad city. The Firefly algorithm was used to optimize the parameters of the SVM to improve its prediction accuracy. The quantitative statistical performance evaluation measures (absolute proportional error (MAPE)) were used to evaluate the performance of the optimization methods. The results proved that the modification method was more accurate compared to the basic method and PSO-SVM.
\end{abstract}

Keywords-SVM; FA; Load forecasting; PSO

\section{INTRODUCTION}

In the context of increasing power demand and development of the power market, load forecasting is a major challenge in terms of power system planning and operation [13]. The advantage of a precise load forecasting is to help the operators make decisions for the commitment unit, reduce the reserve capacity, and make a proper schedule for maintenance planning [4]. Apart from holiday forecasting, the power system experts have divided forecasting into long, medium, and shortterm forecasting based on the duration of the term.

The intelligent methods of power system forecasting have been increased significantly over the last decades to reduce the error in the expected power system changes [5-9]. The Autoregressive Moving Average (ARMA) with the Artificial Neural Network (ANN) is accurate in predicting small power and depends only on the data [10]. Jie Shi has applied a hybrid forecasting model based on grey relational analysis and wind speed distribution features [3]. Many solutions have been introduced in electrical problems since 2008 when the Firefly algorithm (FA) was proposed by Xin-She Yang [11]. Bayesian Neural Networks, Deep neural networks, and Deep neural networks have been assumed as a feasible method for presaging the power in this year [7-9].

From the other hand, the SVM is a regression technique which was feigned by Vladimir Vapnik in 1995 [11]. Since its development, its usage in forecasting has increased gradually [11]. Xing et al suggested many hybrid prediction methods based on SVM, and one of the methods depend on the use of least squares support vector machine [12]. The PSO-SVM was developed by many researchers such as: Nadtoka I for short forecasting [13, 14].

However, in addition to the non-suitable parameters for SVM method, there is no powerful method yet in the area of short-term prediction [15]. This problem is because the SVM accuracy depends on the suitable values of two parameters the Lagrange multipliers ( $\alpha$ ) and the regularization parameter $\left.{ }_{(}^{C}\right)[16]$.

In this paper, a new method which depends on two methods (Firefly optimization and SVM (FA-SVM)) was used to predict the power load of Bagdad city. Firefly optimization was used to optimize $\alpha$ and $C$ parameters to ensure an optimal load forecasting. This forecasting was performed using one-year real data (power and weather) of Baghdad and the performance of the proposed method on the forecasting accuracy was compared to that of two forecasting methods. For each iteration of SVM, many iterations were performed to select the values of $\alpha$ and $C$. A comparison of the absolute proportional error between SVM, PSO-SVM, and the proposed methods was performed, and the results showed that the proposed method was more efficient in prediction compared to the other methods in terms of forecasting the system within a short duration.

\section{THEORETICAL BACKGROUND}

\section{A. SVM}

Basically, any learning method depends on a driven data which is based on the multi-disciplinary ideas that combine with the facility of a computer to perform an expected action on other datasets [16]. Fig. 1 provides a schematic flowchart of any simple learning model [17].

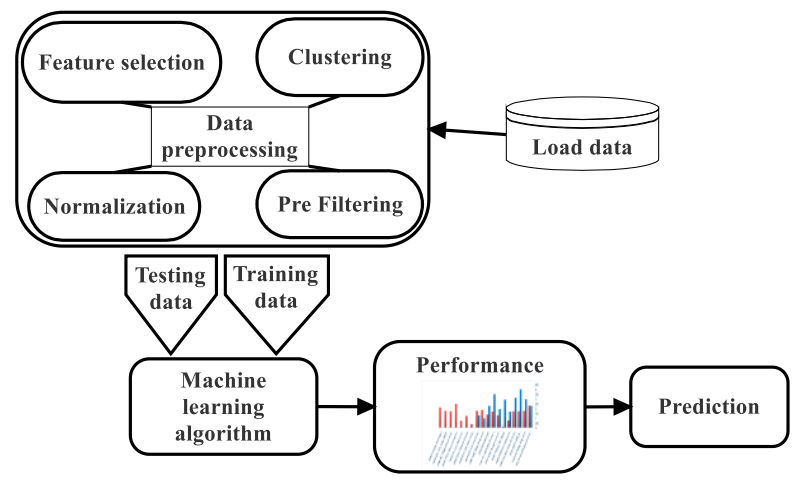

Fig. 1. Machine Learning basic Model. 
Vladimir proposed one of the most famous artificial intelligent learning machines and named it as Support Vector Machines (SVM) [18]. Basically, the SVM optimization equations can be expressed as [13]:

$$
\min J=\frac{1}{2}\|\omega\|^{2}+\frac{1}{2} C \sum_{i=1}^{l} e_{i}^{2}-\sum_{i=1}^{l} \alpha_{i}\left(\omega^{T} \varphi\left(x_{i}\right)+b+e_{i}-y_{i}\right)
$$

Where:- $e_{i}$ is the initial error, $e \in R^{l \times 1}$ is the vector error, $C$ is the regularization parameter, $\alpha$ are the Lagrange multipliers, $\alpha \in R^{l \times 1}$. Also, the nonlinear forecasting model can be expressed as [13]:

$$
P_{i+1}=\sum_{i=1}^{m} \alpha_{i} K\left(x_{i}, x\right)+b
$$

Where:- $i=1, \ldots, m ; x_{i}$ is the input vector, $P(t)=$ power, $T(t)=$ temperature, and $L(t)=$ natural illumination; $x$ $=$ coordinates of the center of the scattering vector; $\alpha_{i}, b=$ linear coefficients; $m=$ dimension of the input vector; $K\left(x_{i}, x\right)=$ Kernel function which performs a nonlinear mapping of the input data space.

\section{B. Firefly Algorithm}

The flashing behavior of fireflies inspired Xin-She Yang to develop a novel swarm-intelligence-based method of optimization in 2008 [19]. The light signal from a firefly serves as a signal that attracts the other fireflies towards itself. Mathematically, all the fireflies in the FA are assumed to be unisex and can be attracted to each order based on the intensity of the emitted light from each firefly [20]. The objective function is the leader of any combination of the two brightest fireflies in one population on every algorithmic iteration [21].

As a firefly's attractiveness is proportional to the light intensity which is noticed by adjacent fireflies, the variation of attractiveness with the distance $r$ can be described in terms of the attractiveness at $r$ by [22]:

$\beta=\beta_{o} e^{-8 r^{2}}$

The change in the position of firefly $i$ which is attracted to firefly $j$ is calculated by [22]:

$x_{i}^{t+1}=x_{i}^{t} \beta=\beta_{o} e^{-\gamma r_{i, j}^{2}}\left(x_{j}^{t}-x_{i}^{t}\right) \alpha_{t} \epsilon_{i}^{t}+\pi r^{2}$

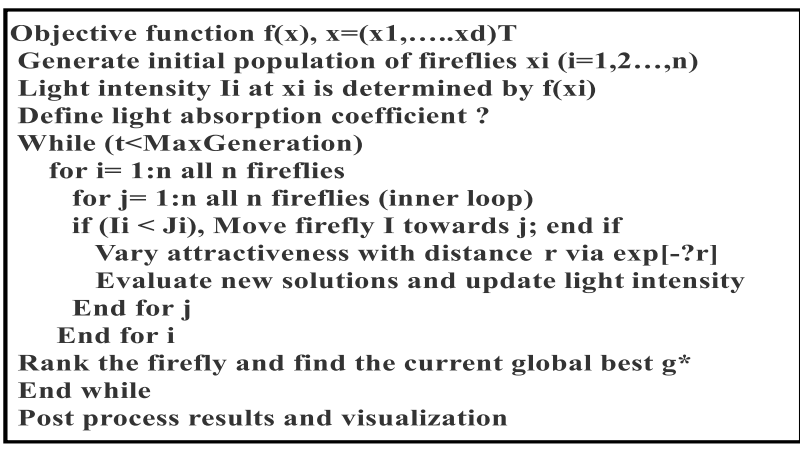

Fig. 2. Firefly Algorithm.
Where:- $\epsilon_{i}^{t}$ is a vector of random numbers on time $t$, which depends on Gaussian and The third term is randomization with $\alpha_{t}$. The firefly algorithm is as shown in Fig 2 [18].

\section{THE PROPOSED METHOD}

One of the main challenges in Baghdad load forecasting is the low accuracy of prediction due to the high complexity of the power system and the climate data. Also, the accuracy of SVM forecasting depends on two parameters which are the regularization parameter and the Lagrange multipliers. These parameters represent the degree of correlation on the SV learning specimen input spaces. Therefore, a new hybrid approach based on SVM and FA is introduced in this study to deal with these two issues. The employment and selection of the optimal values of the two parameters of SVM $(\sigma, C)$ were based on the FA algorithm. Normally, any evolutionary algorithm requires a fitness function which will serve as the optimization objective [15]. In the area of forecasting, there are many criteria-based methods to gauge the accuracy of a system, such as the relative percentage error, the mean absolute percentage error, the root mean square error, and the absolute proportional error which is selected to gauge the proposed method. The proposed method is shown in the following algorithm:

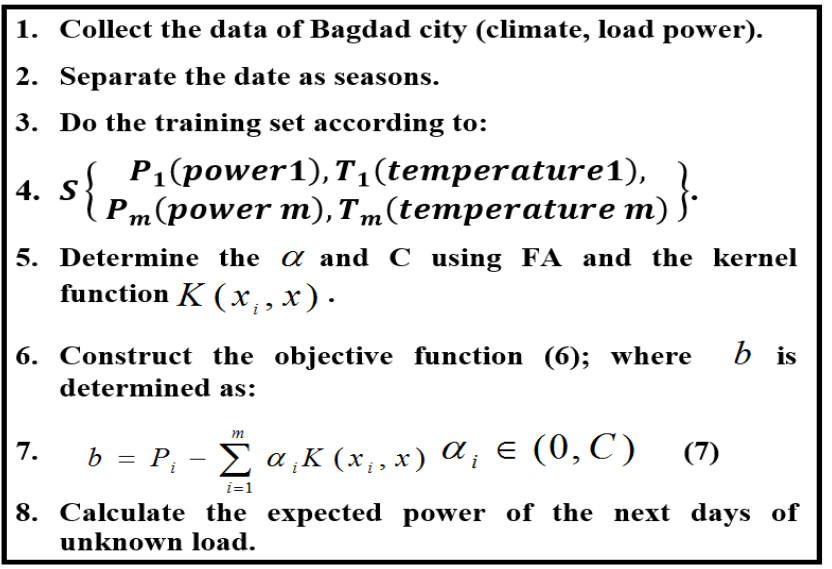

The flow chart of the FA-SVM for the mid-season is shown in Fig. 3.

The selected limits of the initial values of $\mathrm{C}$ and $\sigma$ are $[0.1,250]$ and $[0.1,15]$, respectively, $m=25$. The main FA parameters are set to the optimal settings $\beta 0=0.25, \gamma=1.0$, $\alpha=0.3$, and the number of Fireflies $=25$, number of iterations $=80$. For the PSO, the bird step $=25, \omega=0.7$; acceleration constants $c_{1}$ and $c_{2}=1.8$ as earlier recommended by [13].

The real data of Bagdad city was taken for the period of $1^{\text {st }}$ March $2017-31^{\text {st }}$ December 2017 and used for the calculation of daily forecasts using the three compared methods. The power data was obtained from the Iraqi national dispatch center, while the climate data were obtained from the Iraqi meteorological organization and seismology. the comparison between the methods depended on the prediction errors (mean absolute error percentage mape) calculated as follows:

MAPE $=\frac{1}{24 \cdot N} \cdot \sum_{i=1}^{24} \sum_{k=1}^{N} \frac{\left|P_{i, k}^{\text {forcast }}-P_{i, k}^{\text {actual }}\right|}{P_{i, k}^{\text {actual }}} \cdot 100 \%$ 


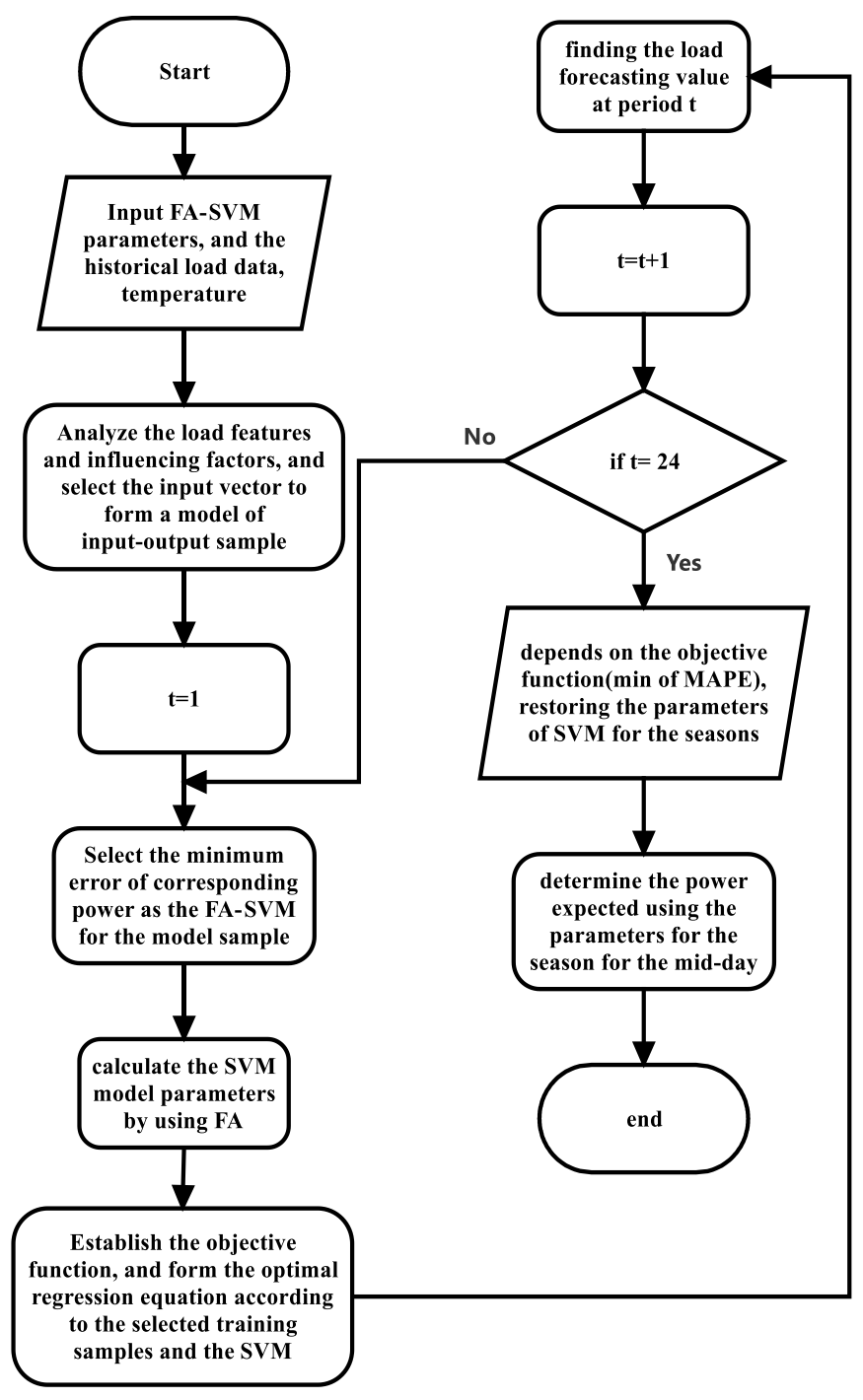

Fig. 3. FA-SVM Flowchart.

\section{SimULATION RESULTS}

The aim of the work is to examine the capability of the proposed FA-SVM in reducing the mean absolute error percentage (MAPE) in high complex prediction problems. The simulation was conducted in a MATLAB platform for the three algorithms during the forecast of the power load of Baghdad city. The SVM was first used before using the hybrid technique (PSO-SVM) to select SVM parameters. Finally, the FA was used to optimize the selected SVM parameters. The prediction results were compared with the real data based on the mean absolute error percentage. Figs. 4-7 showed the real data and forecasted load for a single day in spring, summer, autumn, and winter seasons. From these Figs, it was evident that the proposed method achieved results that closely matched the real data.

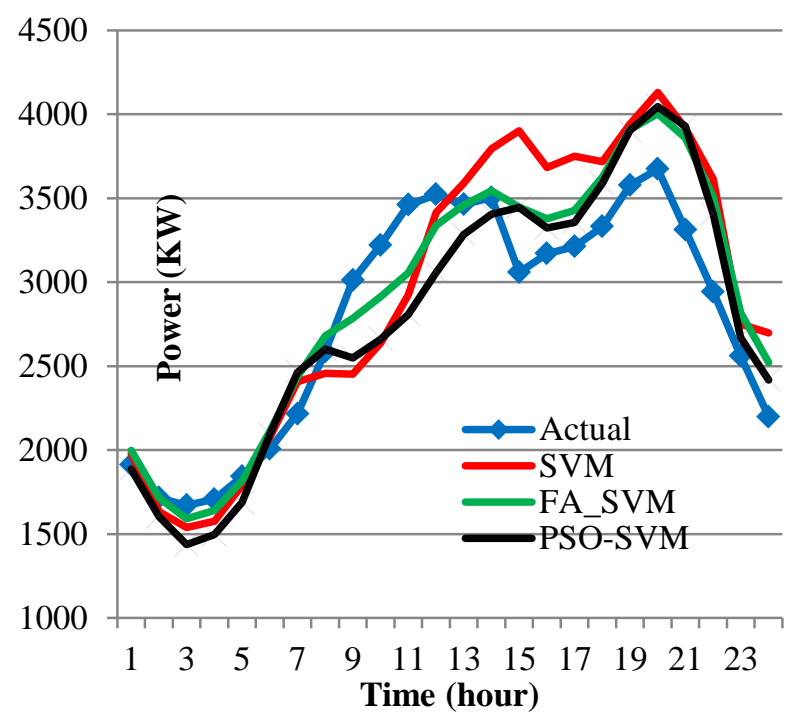

Fig. 4. Daily Forecasting Load Curve of Mid-Spring.

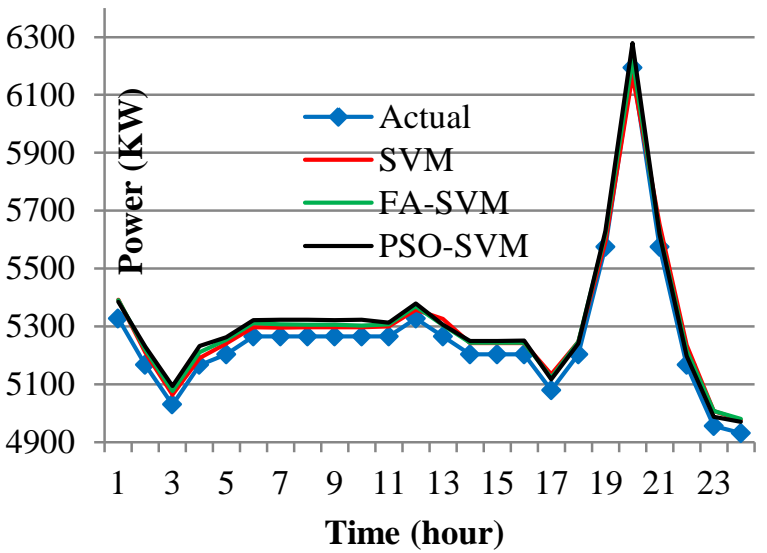

Fig. 5. Daily Forecasting Load Curve of Mid-Autumn.

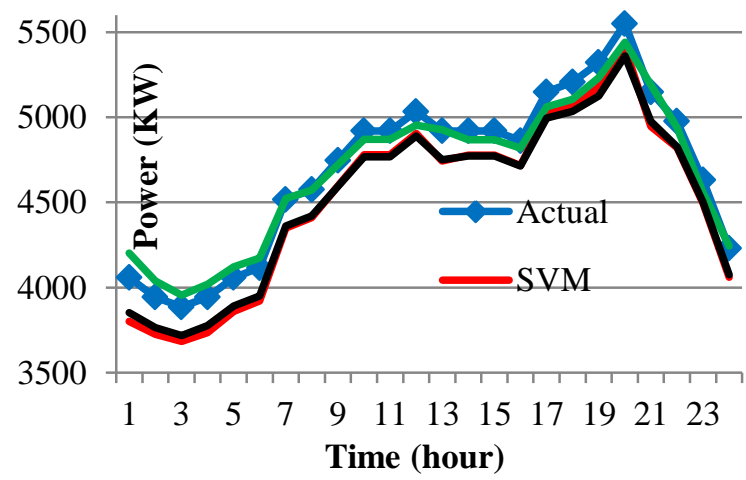

Fig. 6. Daily Forecasting Load Curve of Mid-Summer. 


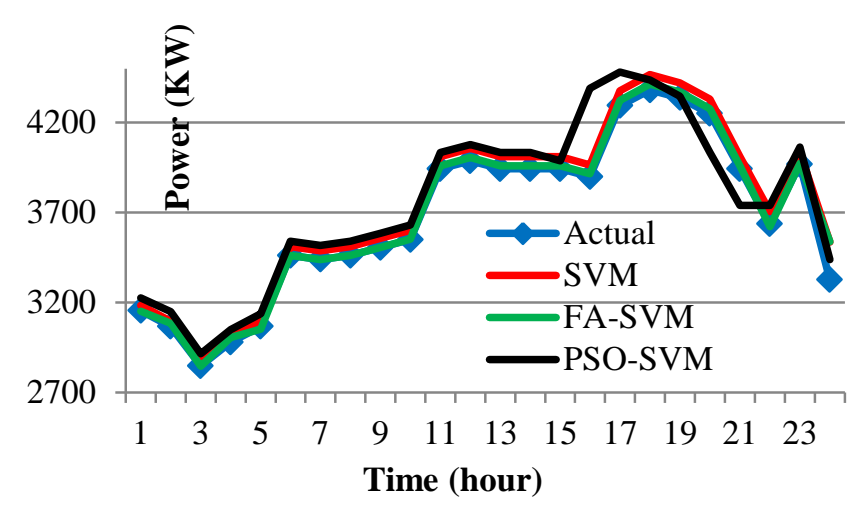

Fig. 7. Daily Forecasting Load Curve of Mid-Winter.

TABLE I. MAPE FOR SVM

\begin{tabular}{|c|c|c|c|c|}
\hline \multicolumn{5}{|l|}{ SVM } \\
\hline date & $21-32017$ & $21-92017$ & $21-6-2017$ & $21-122017$ \\
\hline $\mathrm{C}$ & 30 & 30 & 30 & 30 \\
\hline$\sigma$ & 2 & 2 & 2 & 2 \\
\hline MAPE $\%$ & 4.55 & 0.8 & 3.65 & 1.76 \\
\hline \multicolumn{5}{|c|}{ Overall (MAPE) $2.69 \%$} \\
\hline
\end{tabular}

TABLE II. MAPE FOR PSO-SVM

\begin{tabular}{|l|l|l|l|l|}
\hline \multicolumn{5}{|l|}{ PSO-SVM } \\
\hline date & $21-32017$ & $\begin{array}{r}21-9 \\
2017\end{array}$ & $21-62017$ & $21-122017$ \\
\hline $\mathrm{C}$ & 2.7 & 180 & 14 & 205 \\
\hline$\sigma$ & 7.011 & 2.54 & 2.64 & 10.12 \\
\hline MAPE\% & 4.5 & 0.8 & 3.51 & 1.39 \\
\hline Overall (MAPE) $2.55 \%$ & & \\
\hline
\end{tabular}

TABLE III. MAPE FOR FA-SVM

\begin{tabular}{|l|l|l|l|l|}
\hline \multicolumn{5}{|l|}{ FA-SVM } \\
\hline date & $21-32017$ & $21-92017$ & $21-62017$ & $21-12-2017$ \\
\hline $\mathrm{C}$ & 2.0601 & 150.0000 & 2.9746 & 149.9249 \\
\hline$\sigma$ & 6.4242 & 2.7100 & 0.1000 & 0.7775 \\
\hline MAPE\% & 3.61 & 0.75 & 1.27 & 0.62 \\
\hline Overall (MAPE) $1.5875 \%$ & & & \\
\hline
\end{tabular}

Tables I, II, and III show the results of percentage error be for different days and methods. Form this tables, it can be concluded that FA-SVR has more ability to reach the load characteristics than SVM and PSO-SVM. Also, the tables are explaining the selected values of VSM parameters for each method.

\section{CONCLUSION}

An SVM-FA hybrid algorithm was proposed in this study for the forecast of the daily load power of Baghdad city for the period of $1^{\text {st }}$ March $2017-31^{\text {st }}$ December 2017. This data covered the four available seasons of the year in Iraqi. In this proposed method, the FA was employed to adjust the values of the SVM model parameters in order to achieve more promising data. The results obtained from the proposed SVM-FA method showed an acceptable percentage error margin with the real data. The accuracy of the methods was verified and compared using the absolute proportional error as a criterion.

\section{REFERENCES}

[1] Q. Wu, "Product demand forecasts using wavelet kernel support vector machine and particle swarm optimization in manufacture system," J. Comput. Appl. Math., vol. 233, no. 10, pp. 2481-2491, 2010.

[2] G. Sreenivasan, "PSO Based Short-Term Hydrothermal Scheduling with Prohibited Discharge Zones," IJACSA - Int. J. Adv. Comput. Sci. Appl., vol. 2, no. 9, pp. 97-105, 2011.

[3] J. Shi, Z. Ding, W. Lee, Y. Yang, Y. Liu, and M. Zhang, "Hybrid Forecasting Model for Very-Short Term Wind Power Forecasting Based on Grey Relational Analysis and Wind Speed Distribution Features," IEEE Trans. Smart Grid, vol. 5, no. 1, pp. 521-526, 2014.

[4] C.-M. Huang, C.-J. Kuo, and Y.-C. Huang, "Short-term wind power forecasting and uncertainty analysis using a hybrid intelligent method," IET Renew. Power Gener., vol. 11, no. 5, pp. 678-687, 2017.

[5] Y. Jiang, X. Chen, K. Yu, and Y. Liao, "Short-term wind power forecasting using hybrid method based on enhanced boosting algorithm," J. Mod. Power Syst. Clean Energy, vol. 5, no. 1, pp. 126133, 2017.

[6] J. Shi et al., "Hybrid Forecasting Model for Very-Short Term Wind Power Forecasting Based on Grey Relational Analysis and Wind Speed Distribution Features," vol. 5, no. 1, pp. 521-526, 2014.

[7] S. Zheng, Q. Zhong, L. Peng, and X. Chai, "A Simple Method of Residential Electricity Load Forecasting by Improved Bayesian Neural Networks," Math. Probl. Eng., vol. 2018, 2018.

[8] M. Easley, L. Haney, J. Paul, K. Fowler, and H. Wu, "Deep neural networks for short-term load forecasting in ERCOT system," in 2018 IEEE Texas Power and Energy Conference, TPEC 2018, 2018, vol. 2018-Febru, pp. 1-6.

[9] T. Vantuch, A. G. Vidal, A. P. Ramallo-Gonzalez, A. F. Skarmeta, and S. Misak, "Machine learning based electric load forecasting for short and long-term period," in IEEE World Forum on Internet of Things, WF-IoT 2018 - Proceedings, 2018, vol. 2018-Janua, pp. 511-516.

[10] S. Zhang, Y. Wang, M. Liu, and Z. Bao, "Data-Based Line Trip Fault Prediction in Power Systems Using LSTM Networks and SVM," IEEE Access, vol. 6, pp. 7675-7686, 2017.

[11] P. Vähäkyla, E. Hakonen, and P. Léman, "Short-term forecasting of grid load using Box-Jenkins techniques," Int. J. Electr. Power Energy Syst., vol. 2, no. 1, pp. 29-34, 1980.

[12] W. H. NIU Dong-xiao, GU Zhi-hong, XING Mian, "Study on Forecasting Approach to Short-term Load of SVM Based on Data Mining," in Proceedings of the CSEE, 2006, pp. 1-6.

[13] I. I. Nadtoka and M. Al-Zihery Balasim, "Mathematical modelling and short-term forecasting of electricity consumption of the power system, with due account of air temperature and natural illumination, based on support vector machine and particle swarm," in Procedia Engineering, 2015, vol. 129, pp. 657-663.

[14] G. Ren, S. Wen, Z. Yan, R. Hu, Z. Zeng, and Y. Cao, "Power Load 
Forecasting Based on Support Vector Machine and Particle Swarm Optimization," no. 1, 2016.

[15] A. Kavousi-Fard, H. Samet, and F. Marzbani, "A new hybrid Modified Firefly Algorithm and Support Vector Regression model for accurate Short Term Load Forecasting," Expert Syst. Appl., vol. 41, no. 13, pp. 6047-6056, 2014.

[16] L. Demidova, E. Nikulchev, and Y. Sokolova, "The SVM Classifier Based on the Modified Particle Swarm Optimization," Int. J. Adv. Comput. Sci. Appl., vol. 7, no. 2, 2016.

[17] S. N. Fallah, R. C. Deo, M. Shojafar, M. Conti, and S. Shamshirband, "Computational intelligence approaches for energy load forecasting in smart energy management grids: State of the art, future challenges, and research directions," Energies, vol. 11, no. 3, 2018.
[18] S. Dai, D. Niu, and Y. Han, "Forecasting of Power Grid Investment in China Based on Support Vector Machine Optimized by Differential Evolution Algorithm and GreyWolf Optimization Algorithm," Appl. Sci., vol. 8, no. 636, pp. 1-19, 2018.

[19] I. Fister, X. S. Yang, and J. Brest, "On the randomized firefly algorithm,” Stud. Comput. Intell., vol. 516, pp. 27-48, 2014.

[20] X. S. Yang and X. He, "Firefly algorithm: recent advances and applications," Int. J. Swarm Intell., vol. 1, no. 1, p. 36, 2013.

[21] N. Ali, M. A. Othman, M. N. Husain, and M. H. Misran, "A review of firefly algorithm,” ARPN J. Eng. Appl. Sci., vol. 9, no. 10, pp. 1732 1736, 2014.

[22] A. S. Jaber, "A Novel Tuning Method of PID Controller for a BLDC Motor Based on Segmentation of Firefly Algorithm," Indian J. Sci. Technol., vol. 10, no. 6, 2017. 